\title{
Views on Precision Medicine among Health Professionals in Korea: A Mixed Methods Study
}

\author{
Ha Na Cho ${ }^{1}$, Soo-Yong Shin ${ }^{2}$, Bin Hwangbo ${ }^{3}$, Yoon Jung Chang ${ }^{4}$, \\ Juhee $\mathrm{Cho}^{5}$, Sun-Young Kong ${ }^{6}$, Kui Son $\mathrm{Choi}^{1}$, and Eun Sook Lee ${ }^{7}$ \\ ${ }^{1}$ Graduate School of Cancer Science and Policy, National Cancer Center, Goyang; \\ ${ }^{2}$ Department of Digital Health, Samsung Medical Center, Sungkyunkwan University School of Medicine, Seoul; \\ ${ }^{3}$ Center for Lung Cancer, Research Institute and Hospital, National Cancer Center, Goyang; \\ ${ }^{4}$ National Cancer Control Institute, National Cancer Center, Goyang; \\ ${ }^{5}$ Center for Clinical Epidemiology, Samsung Medical Center, Sungkyunkwan University School of Medicine, Seoul; \\ ${ }^{6}$ Center for Diagnostic Oncology, Research Institute and Hospital, National Cancer Center, Goyang; \\ ${ }^{7}$ Center for Breast Cancer, Research Institute and Hospital, National Cancer Center, Goyang, Korea.
}

\begin{abstract}
This study aimed to investigate awareness, attitudes, and perspectives on precision medicine among health professionals in Korea and to identify issues that need to be addressed before implementing precision medicine. Mixed methods research was applied. For qualitative research, a semi-structured focus group interview was conducted with six health professionals. For quantitative research, a self-reported survey was administered. A total of 542 health professionals participated in the survey, and 526 completed the entire questionnaire. Health professionals showed positive attitudes toward precision medicine. About 95-96\% of respondents agreed that precision medicine will be effective in treatment and precise diagnosis, and $69.9 \%$ reported that they would participate as study subjects. Meanwhile, they expressed concerns regarding educating patients and health professionals in precision medicine and developing research and data sharing infrastructure. Also, they emphasized the importance of developing precision medicine in an equitable way. Despite varying levels of awareness of precision medicine, the health professionals expressed a willingness to engage in precision medicine research, and recommended that health professionals work closely with policymakers to design precision medicine in a way that can be effectively adopted. Health professionals showed had a positive, but cautious, attitude toward precision medicine. The results of this study suggest areas to be addressed before ushering in precision medicine in Korea.
\end{abstract}

Key Words: Precision medicine, mixed method, awareness, attitude, neoplasms

Precision medicine seeks to achieve disease prevention and treatment that is optimized to individual characteristics for improved treatment outcomes and quality of life. ${ }^{1-3}$ Rapid devel-

Received: September 9, 2019 Revised: December 21, 2019

Accepted: December 27, 2019

Co-corresponding authors: Kui Son Choi, PhD, Graduate School of Cancer Science and Policy, National Cancer Center, 323 IIsan-ro, Ilsandong-gu, Goyang 10408, Korea. Tel: 82-31-920-2912, Fax: 82-31-920-2189, E-mail: kschoi@ncc.re.kr and Eun Sook Lee, MD, PhD, Center for Breast Cancer, Research Institute and Hospital, National Cancer Center, 323 Ilsan-ro, Ilsandong-gu, Goyang 10408, Korea. Tel: 82-31-920-1633, Fax: 82-31-920-2189, E-mail: eslee@ncc.re.kr

-The authors have no potential conflicts of interest to disclose.

(C) Copyright: Yonsei University College of Medicine 2020

This is an Open Access article distributed under the terms of the Creative Commons Attribution Non-Commercial License (https://creativecommons.org/licenses/ by-nc/4.0) which permits unrestricted non-commercial use, distribution, and reproduction in any medium, provided the original work is properly cited. opment of biomedical technology and significant reductions in the cost of genome sequencing have helped in realizing precision medicine in medical practice. ${ }^{4}$ With the announcement of the Precision Medicine Initiative by President Obama in $2015,{ }^{1,5}$ the US and other countries, including the UK, ${ }^{6}$ France, ${ }^{7}$ Japan, ${ }^{8}$ China, ${ }^{9}$ and Korea, ${ }^{10,11}$ have been working on developing precision medicine as a new medical paradigm to improve the accuracy and efficacy of medicine globally.

As views on and understanding of precision medicine among health professionals might play an important role in the realization of precision medicine, we aimed to investigate awareness, attitudes, and perspectives on precision medicine in Korea via mixed methods study. Initially, we conducted a qualitative study to explore awareness, attitudes, expectations, and concerns regarding precision medicine among health profession- 
als. We then conducted a quantitative study to expand and generalize the qualitative findings.

In the current study, we conducted mixed methods research, which involves collecting, analyzing, and integrating quantitative and qualitative research. ${ }^{12}$ We adopted a sequential exploratory design comprising an initial phase of qualitative data collection and analysis, followed by a phase of quantitative data collection and analysis, to explore opinions on precision medicine. In the initial phase, a semi-structured focus group interview (FGI) was conducted. To begin, we sent an invitation letter to the members of the Big Data Research Advisory Committee via e-mail. Those who accepted our invitation helped us identify experts on precision medicine that might want to participate in the study. Through such snowball sampling, we were able to recruit six experts who are working in the areas of preventive medicine, bioinformatics or health data sciences, oncology, and big data research. The FGI was conducted on October 12, 2016. All participants were asked to provide written informed consent prior to participation in the interviews. An FGI guide, which included questions designed to ascertain their perspectives on precision medicine, potential challenges that may impede or delay the realization of precision medicine, and recommendations for the adoption of precision medicine, was used. The interviews were audio recorded and transcribed verbatim. Using a qualitative content analysis approach, the transcripts were analyzed manually by two experts. Thematic analysis was conducted, then the substantial content of the dialogue was extracted, coded, and classified according to the over-reaching themes. During these processes, two experts cross-checked and a third senior researcher finally reviewed coded data confirming similarities in themes.

In the second phase, utilizing our results from the qualitative research, we developed a survey questionnaire and administered a self-reported survey (Supplementary Materials, only online). Health professionals, including clinicians, nurses, professors, and researchers, were then recruited during the 21st Annual Fall Symposium of the Korean Cancer Association held on November 18, 2016. All participants were asked to provide written informed consent prior to participation in the surveys. A total of 542 health professionals participated in the survey, and 526 completed the entire questionnaire. The questionnaire was developed to assess awareness and attitudes about precision medicine. We conducted descriptive analysis and chi-square tests to compare the distributions of awareness and attitudes about precision medicine according to job type. We further conducted post-hoc analysis for pairwise comparisons with Bonferroni correction of the $p$ values. We categorized health professions into three different groups: 1) clinicians, 2) researchers, and 3) other health care professionals. All statistical analyses were conducted using SAS software, version 9.3 (SAS Institute, Cary, NC, USA). The study procedure was approved by the Institutional Review Board of the National Cancer Center, Korea (NCC-20160256).
When asked about the main application areas for precision medicine, most participants reported that it can be widely used in patient treatment: "The major use of precision medicine would be in facilitating the use of personalized drugs for each patient." Some participants showed high expectations for precision medicine. They believed precision medicine could improve the quality of life of cancer patients by avoiding side effects: "In cancer treatment, adverse drug reactions are quite large. However, if we find out a medicine that directly targets an individual disease, we will be able to avoid all unsuccessful attempts to find the right treatment without having to deal with unwanted side effects." Further, they expected that precision medicine would be reduce medical costs in the long term: "If ineffective treatments are avoided, we can reduce side effects and save on medical costs." However, some participants also confessed that the concept of precision medicine and its possible practical implications are still unclear, except in the field of cancer.

Most participants professed concerns that precision medicine may be too expensive. They expected that only a few patients would be able to access precision medicine, introducing another source of disparity in health care: "In general, new, targeted drugs that are launched on the market are so highlypriced that patients can hardly afford them, unless their cost is fully covered by health insurance." "New technology is always costly. Precision medicine will also be provided at a high price, and only a few people who can afford it will benefit from precision medicine." Also, several participants expressed skepticism regarding the abilities of individual patients to understand precision medicine. They noted that patients often struggle to understand basic medical information: "People do not understand information well enough. They are not ready to learn and understand their genetic profile." Further, some participants worried about a lack of competence among physicians: "When physicians perform DNA sequencing, they should explain the results to the patients, but some of them do not know about the genome." Some experts expressed concerns that use of incomplete and unstandardized data may lead confusion for both the physician and patients and thus hinder the implementation of precision medicine. In this context, they pointed out the lack of bioinformaticians and biomedical informatics specialists: "Precision medicine is based on IT infrastructure.... There are many IT personnel in Korea, but biotechnicians in the medical field are too scarce in Korea."

Of the 526 health professions who completed the survey, 187 were male (35.6\%) and 339 were female (64.4\%), with a mean age of 35.2 years. Health professionals were categorized as clinicians (21.5\%), researchers (37.6\%), and other health professionals (40.9\%) (Table 1). Most of the participants described precision medicine as personalized medicine (41.6\%); precise/ detailed/specific treatment (11.4\%); diagnosis testing (11.2\%); gene therapy, gene analysis, or genetic testing (6.8\%); cancer treatment, cancer diagnosis, or cancer prevention (2.7\%); ex- 
pensive (1.7\%); and effective treatment with fewer side-effects $(1.3 \%)$ (Table 2). To identify the core information needed to adopt precision medicine into practice, the highest proportion of respondents (89.2\%) agreed that genetic information is needed: in particular, researchers were more likely to answer that genetic information is needed than other health professionals ( $p=0.041)$. Also, the respondents indicated that genetic and clinical information is needed more than health behavior information.

About 96\% of the respondents reported that precision medicine will be effective in patient treatment and will provide precise diagnosis (94.9\%) (Table 3). However, overall expectations on precision medicine were the lowest among clinicians, compared with other health care professionals. Specifically, expec-

Table 1. Demographic Characteristics According to Different Types of Health Professionals

\begin{tabular}{|c|c|c|c|c|c|}
\hline & $\begin{array}{l}\text { Overall } \\
(n=526)\end{array}$ & $\begin{array}{c}\text { Clinicians } \\
(n=113)\end{array}$ & $\begin{array}{l}\text { Researchers } \\
(n=198)\end{array}$ & $\begin{array}{l}\text { Other health } \\
\text { professionals ( } n=215)\end{array}$ & $p$ value \\
\hline Sex, $n(\%)$ & & & & & $<0.001^{\text {tł }}$ \\
\hline Male & $187(35.6)$ & $59(52.2)$ & $62(31.3)$ & $66(30.7)$ & \\
\hline Female & $339(64.4)$ & $54(47.8)$ & $136(68.7)$ & $149(69.3)$ & \\
\hline Age, $n(\%)$ & & & & & $<0.001^{1 \ddagger \S}$ \\
\hline $20-30$ & $373(70.9)$ & $53(46.9)$ & $138(69.7)$ & $182(84.7)$ & \\
\hline$\geq 40$ & $153(29.1)$ & $60(53.1)$ & $60(30.3)$ & $33(15.3)$ & \\
\hline Education*, n (\%) & & & & & $<0.001^{\ddagger \S}$ \\
\hline University & $190(36.5)$ & $15(13.3)$ & $31(15.7)$ & $144(68.9)$ & \\
\hline Master's degree and above & $330(63.5)$ & $98(86.7)$ & $167(84.3)$ & $65(31.1)$ & \\
\hline Workplace, n (\%) & & & & & $<0.001^{1 \neq \$}$ \\
\hline Hospital & $217(41.3)$ & $98(86.7)$ & 74 (37.4) & $45(20.9)$ & \\
\hline University & $77(14.6)$ & $2(1.8)$ & $32(16.1)$ & $43(20.0)$ & \\
\hline Research institute & $93(17.7)$ & $11(9.7)$ & $55(27.8)$ & $27(12.6)$ & \\
\hline Pharmaceutical company and others & $139(26.4)$ & $2(1.8)$ & $37(18.7)$ & $100(46.5)$ & \\
\hline
\end{tabular}

*Excluded six without answers, ${ }^{\dagger}$ Statistically different between clinicians and researchers after Bonferroni correction ( $\left.p<0.001\right)$, ${ }^{\ddagger}$ Statistically different between clinicians and other health professionals after Bonferroni correction $(p<0.001)$, ${ }^{5}$ Statistically different between researchers and other health professionals after Bonferroni correction ( $p<0.001)$.

Table 2. Words that Explain Precision Medicine and Information Required for Precision Medicine

\begin{tabular}{|c|c|c|c|c|c|}
\hline & $\begin{array}{l}\text { Overall } \\
(n=526)\end{array}$ & $\begin{array}{c}\text { Clinicians } \\
(n=113)\end{array}$ & $\begin{array}{l}\text { Researchers } \\
(n=198)\end{array}$ & $\begin{array}{c}\text { Other health } \\
\text { professionals }(n=215)\end{array}$ & $p$ value \\
\hline \multicolumn{6}{|c|}{ What comes to mind when you think of precision medicine (open-ended questions), $\mathrm{n}(\%)$} \\
\hline Personalized medicine & $219(41.6)$ & $43(38.1)$ & $101(51.0)$ & $75(34.9)$ & \\
\hline Precise/detailed/specific test & $60(11.4)$ & $4(3.5)$ & $26(13.1)$ & $30(14.0)$ & \\
\hline Treatment/diagnosis/test & $59(11.2)$ & $14(12.4)$ & $15(7.6)$ & $30(14.0)$ & \\
\hline Gene therapy/analysis, genetic test & $36(6.8)$ & $14(12.4)$ & $11(5.6)$ & $11(5.1)$ & \\
\hline Cancer treatment/diagnosis/prevention & $14(2.7)$ & $4(3.5)$ & $6(3.0)$ & $4(1.9)$ & \\
\hline Expensive & $9(1.7)$ & $3(2.7)$ & $2(1.0)$ & $4(1.9)$ & \\
\hline Effective/low side-effects & $7(1.3)$ & $2(1.8)$ & $0(0.0)$ & $5(2.3)$ & \\
\hline Etc. & $117(22.2)$ & $26(23.0)$ & $36(18.2)$ & $55(25.6)$ & \\
\hline No answer & $5(1.0)$ & $3(2.7)$ & $1(0.5)$ & $1(0.5)$ & \\
\hline \multicolumn{6}{|c|}{ Information required for precision medicine (multiple responses), n (\%) } \\
\hline Genetic information & $469(89.2)$ & $104(92.0)$ & $183(92.4)$ & $182(84.7)$ & $0.022^{*}$ \\
\hline Bio specimen (blood, tissue, cell) & $393(74.7)$ & $96(85.0)$ & $149(75.3)$ & $148(68.8)$ & $0.006^{\dagger}$ \\
\hline Body measurement (height, weight) & $243(46.2)$ & $61(54.0)$ & $87(43.9)$ & $95(44.2)$ & 0.173 \\
\hline Medical records & $392(74.5)$ & $82(72.6)$ & $156(78.8)$ & $154(71.6)$ & 0.215 \\
\hline Lifestyle & $249(47.3)$ & $54(47.8)$ & $91(46.0)$ & $104(48.4)$ & 0.882 \\
\hline Health insurance claims data & $88(16.7)$ & $26(23.0)$ & $26(13.1)$ & $36(16.7)$ & 0.081 \\
\hline Income & $87(16.5)$ & $26(23.0)$ & $27(13.6)$ & $34(15.8)$ & 0.095 \\
\hline Residential area of the patient & $84(16.0)$ & $21(18.6)$ & $33(16.7)$ & $30(14.0)$ & 0.522 \\
\hline Economic consumption behavior & $47(8.9)$ & $17(15.0)$ & $13(6.6)$ & $17(7.9)$ & $0.033^{\ddagger}$ \\
\hline
\end{tabular}

${ }^{*}$ Statistically different between researchers and other health professionals with after Bonferroni correction $(p=0.041)$, ${ }^{\dagger}$ Statistically different between clinicians and other health professionals after Bonferroni correction $(p=0.004)$, ${ }^{\ddagger}$ Statistically different between clinicians and researchers after Bonferroni correction $(p=0.045)$. 
tations for improving quality of life, predicting disease in-advance, and reducing unnecessary tests with which to diagnose disease, and providing precise diagnosis were significantly lower among clinicians than the other two groups. Regarding concerns related to precision medicine, health professionals were most concerned about increases in disparity and health care costs. However, concerns for infringing upon personal information were relatively low among clinicians and researchers, compared with other health professionals.

Regarding the respondents' willingness to participate as study subjects in precision medicine, more than half of all health professionals (69.9\%) reported that they would participate as study subjects (Table 4). Also, most of the respondents answered that they would be willing to provide their health information for the purposes of treating themselves (90.9\%). However, only $62.4 \%$ of respondents agreed that they would be willing to provide their health information for the purposes of treating others. Regarding information that they would provide, more than $90 \%$ of health professionals agreed to provide their genetic, physical, and health behavior information for the purposes of treating themselves. In addition, the percentage of respondents who agreed to provide medical records was high (85.7\%). More researchers agreed to provide medical records than other professionals ( $p=0.010$ ). For treating others, only $45.4 \%$ of respondents indicated being willing to provide their medical records. In a question about the maximum amount payable for a genetic test, overall, $27 \%$ of the respondents answered less than $\$ 100$ US. Clinicians were willing to pay higher costs than other groups: Among clinicians, $31 \%$ answered that they would pay more than $\$ 1,000$ US. Only $15.7 \%$ and $16.9 \%$ of researchers and other health professionals, respectively, responded accordingly, and this difference between clinicians and researchers was statistically significant after Bonferroni correction ( $p=0.021)$.

This study was conducted to investigate awareness of and attitudes on precision medicine among health professionals in Korea using mixed methods. In the FGI findings, health professionals indicated that, while precision medicine is needed, ethical and societal challenges may arise in the process of adopting precision medicine in clinical practice, such as training of health workers, educating the general public, providing equal and affordable access thereto, and standardized data use. The participants also recommended allocating specific funding for developing necessary biobanks and data sharing infrastructure currently lacking in Korea. Privacy protection was seen as particularly important when health data are to be shared, and the respondents suggested that public promotion is needed to make people comfortable with sharing their personal health data.

Through our survey, we discovered that many health professionals associated precision medicine with "personalized medicine." While the terms precision medicine and personalized medicine are used interchangeably, they mean different things. Personalized medicine is an old term referring to individualized medicine, whereas precision medicine means effective approaches based on genetic, environmental, and lifestyle factors. ${ }^{13}$ The concept of precision medicine and its possible practical implications remain unclear among health professionals in Korea and need to be addressed.

In the current study, health professionals reported more expectations than concerns for precision medicine, and around

Table 3. Expectations and concerns regarding precision medicine*

\begin{tabular}{|c|c|c|c|c|c|}
\hline & $\begin{array}{l}\text { Overall } \\
(n=526)\end{array}$ & $\begin{array}{c}\text { Clinicians } \\
(\mathrm{n}=113)\end{array}$ & $\begin{array}{l}\text { Researchers } \\
\quad(n=198)\end{array}$ & $\begin{array}{c}\text { Other health } \\
\text { professionals }(n=215)\end{array}$ & $p$ value \\
\hline \multicolumn{6}{|l|}{ Expectations, $\mathrm{n}(\%)$} \\
\hline Improve quality of life through personalized health care $(n=525)$ & $504(96.0)$ & $103(91.2)$ & $189(95.5)$ & $212(99.1)$ & $0.002^{\dagger}$ \\
\hline Predict disease in advance & $469(89.2)$ & $92(81.4)$ & $177(89.4)$ & $200(93.0)$ & $0.006^{\ddagger}$ \\
\hline Avoid unnecessary tests to diagnose disease & $355(67.5)$ & $51(45.1)$ & $143(72.2)$ & $161(74.9)$ & $<0.001^{\S}$ \\
\hline Possible to provide precise diagnosis $(n=525)$ & $498(94.9)$ & $102(90.3)$ & $192(97.0)$ & $204(95.3)$ & $0.034^{\prime \prime}$ \\
\hline Improve treatment performance $(n=523)$ & $503(96.2)$ & $105(94.6)$ & $192(97.5)$ & $206(95.8)$ & 0.424 \\
\hline Reduce overall health care cost $(n=522)$ & $440(84.3)$ & $92(82.9)$ & $162(82.7)$ & $186(86.5)$ & 0.506 \\
\hline Reduce side effects from treatment & $437(83.1)$ & $90(79.7)$ & $163(82.3)$ & $184(85.6)$ & 0.371 \\
\hline Improve life expectancy & $453(86.1)$ & $101(89.4)$ & $173(87.4)$ & $179(83.3)$ & 0.254 \\
\hline \multicolumn{6}{|l|}{ Concerns, n (\%) } \\
\hline Increase individual health care costs & $442(84.0)$ & $92(81.4)$ & $169(85.4)$ & $181(84.2)$ & 0.658 \\
\hline It would take a long time to apply precision medicine in medical practice & $369(70.2)$ & $81(71.7)$ & $139(70.2)$ & $149(69.3)$ & 0.905 \\
\hline Privacy infringement $(n=525)$ & $242(46.1)$ & $36(32.1)$ & $91(46.0)$ & $115(53.5)$ & $0.001^{\pi}$ \\
\hline Increase disparity in public health & $446(84.8)$ & $87(77.0)$ & $170(85.9)$ & $189(87.9)$ & $0.028^{* *}$ \\
\hline
\end{tabular}

${ }^{*}$ A four-point Likert scale [Strongly disagree (1), disagree (2), agree (3), strongly agree (4)] was used. N (\%) respondents who "agree" or "strongly agree," †Statistically different between clinicians and other health professionals after Bonferroni correction ( $p=0.001)$, "Statistically different between clinicians and other health professionals after Bonferroni correction $(p=0.004)$, ${ }^{5}$ Statistically different between clinicians and researcher and between clinicians and other health professionals after Bonferroni correction ( $p<0.001$ ), "Statistically different between clinicians and researchers after Bonferroni correction ( $p=0.037)$, "Statistically different between clinicians and other health professionals after Bonferroni correction $(p=0.001),{ }^{* *}$ Statistically different between clinicians and other health professionals after Bonferroni correction $(p=0.030)$. 
Ha Na Cho, et al.

Table 4. Willingness to Participate as Study Subjects and to Provide Information for Precision Medicine Research

\begin{tabular}{|c|c|c|c|c|c|}
\hline & $\begin{array}{l}\text { Overall } \\
(n=526)\end{array}$ & $\begin{array}{c}\text { Clinicians } \\
(n=113)\end{array}$ & $\begin{array}{l}\text { Researchers } \\
(n=198)\end{array}$ & $\begin{array}{c}\text { Other health } \\
\text { professionals }(\mathrm{n}=215)\end{array}$ & $p$ value \\
\hline \multicolumn{6}{|c|}{ Participation as study subjects ${ }^{*}, \mathrm{n}(\%)$} \\
\hline Yes & $367(69.9)$ & $81(71.7)$ & $142(72.1)$ & $144(67.0)$ & 0.475 \\
\hline No & $158(30.1)$ & $32(28.3)$ & $55(27.9)$ & $71(33.0)$ & \\
\hline \multicolumn{6}{|c|}{ Willingness to provide information } \\
\hline \multicolumn{6}{|c|}{ For the purposes of treating oneself, $n(\%)$} \\
\hline Genetic information & $489(93.0)$ & $104(92.0)$ & $188(95.0)$ & $197(91.6)$ & 0.381 \\
\hline Body measurements & $499(94.9)$ & $106(93.8)$ & $192(97.0)$ & $201(93.5)$ & 0.235 \\
\hline Medical records & $451(85.7)$ & $95(84.1)$ & $181(91.4)$ & $175(81.4)$ & $0.012^{\ddagger}$ \\
\hline Lifestyle & $474(90.1)$ & $99(87.6)$ & $181(91.4)$ & $194(90.2)$ & 0.556 \\
\hline \multicolumn{6}{|c|}{ For the purposes of treating others, $\mathrm{n}(\%)$} \\
\hline Genetic information & $342(65.0)$ & $80(70.8)$ & $132(66.7)$ & $130(60.5)$ & 0.146 \\
\hline Body measurements & $368(70.0)$ & $85(75.2)$ & $140(70.7)$ & $143(66.5)$ & 0.252 \\
\hline Medical records & $239(45.4)$ & $55(48.7)$ & $96(48.5)$ & $88(40.9)$ & 0.225 \\
\hline Lifestyle & $364(69.2)$ & $82(72.6)$ & $141(71.2)$ & $141(65.6)$ & 0.317 \\
\hline \multicolumn{6}{|c|}{ Maximum amount to pay for genetic tests (USD \$), n $(\%)^{\dagger}$} \\
\hline Do not want to pay & $23(4.4)$ & $4(3.6)$ & $5(2.5)$ & $14(6.6)$ & $0.010^{\S}$ \\
\hline$<100$ & $141(27.0)$ & $25(22.3)$ & $56(28.4)$ & $60(28.2)$ & \\
\hline 100-499 & $124(23.8)$ & $18(16.1)$ & $56(28.4)$ & $50(23.5)$ & \\
\hline 500-999 & $132(25.3)$ & $30(26.8)$ & $49(24.9)$ & $53(24.9)$ & \\
\hline$\geq 1000$ & $102(19.5)$ & 35 (31.3) & $31(15.7)$ & $36(16.9)$ & \\
\hline
\end{tabular}

*Excluded one without response, ${ }^{\dagger}$ Excluded four without response, ${ }^{\ddagger}$ Statistically different between researcher and other health professionals after Bonferron correction $(p=0.010)$, ${ }^{\S}$ Statistically different between clinicians and other health professionals after Bonferroni correction $(p=0.021)$.

$70 \%$ of health professionals responded that they would participate as study subjects for precision medicine research. This result is consistent with previous studies that indicated positive attitudes toward precision medicine. ${ }^{14-16}$ However, in comparison of three different groups of health professionals, we found that clinicians showed lower expectations for precision medicine than researchers and other health professionals. Actually, in the FGI, the interviewees suggested that, most clinicians are not ready to provide genetic testing, interpretation, and personalized treatment to patients in their practice. To increase the number of professionals with the necessary expertise to correctly interpret the genomics profiles of patients, several strategies that involve medical curriculum reform, specialist training, and ongoing physician training must be prepared. ${ }^{15,17-19}$

In addition, the participants in the FGI also highlighted needs for education of the general population to facilitate communication and collaborations between doctor and patient. In precision medicine, patients are encouraged to consult with their healthcare provider to determine their treatment options. However, most patients in Korea are not used to participating in their treatment process. Thus, increasing patient understanding of precision medicine and reshaping the health care system such that patients can participate in their care process are important challenges to implementing precision medicine effectively in Korea.

Our FGI and survey results also showed that developing precision medicine in an equitable way is a major issue. Most health professionals expressed concerns that precision medicine may exacerbate heath disparities if only individuals who are more socioeconomically advantaged are able to benefit from it. This is where health professionals may play an important role by working with decision makers to find ways to implement precision medicine fairly and reasonably. The Scottish Medicines Consortium convened clinicians, health economists, and patient representatives to identify and prioritize new medicines and interventions with good value for the money for patients. ${ }^{20}$ Such collaborative efforts may be a good example of how to fairly implement precision medicine into broader clinical practice.

Although this study was the first time health professionals in Korea discussed precision medicine, the study results are not representative of all health professionals' opinions in Korea due to the small number of participants from a specific area (e.g., oncology). Further, many individuals of younger age who are more favorable to new technology participated in the survey. Thus, further research is needed to ensure the representativeness of the results. Also, more work may be needed to investigate how to introduce precision medicine effectively and equitably.

\section{ACKNOWLEDGEMENTS}

This study was supported by a grant from the National R\&D Program for Cancer Control, Ministry of Health and Welfare, 
Republic of Korea (No. 1520240).

\section{AUTHOR CONTRIBUTIONS}

Conceptualization: Bin Hwangbo, Yoon Jung Chang, Juhee Cho, SunYoung Kong, Kui Son Choi, and Eun Sook Lee. Data curation: Ha Na Cho and Kui Son Choi. Formal analysis: Ha Na Cho and Kui Son Choi. Funding acquisition: Sun-Young Kong and Eun Sook Lee. Investigation: Soo-Yong Shin, Bin Hwangbo, Yoon Jung Chang, Juhee Cho, Sun-Young Kong, Kui Son Choi, and Eun Sook Lee. Methodology: Yoon Jung Chang, Juhee Cho, Sun-Young Kong, and Kui Son Choi. Project administration: Ha Na Cho and Sun-Young Kong. Resources: Sun-Young Kong and Eun Sook Lee. Software: Ha Na Cho. Supervision: Sun-Young Kong, Kui Son Choi, and Eun Sook Lee. Validation: Sun-Young Kong and Kui Son Choi. Visualization: Ha Na Cho. Writing_original draft: Ha Na Cho, Sun-Young Kong, and Kui Son Choi. Writing-review \& editing: Ha Na Cho, Sun-Young Kong, and Kui Son Choi. Approval of final manuscript: All authors.

\section{ORCID iDs}

Ha Na Cho Soo-Yong Shin Bin Hwangbo Yoon Jung Chang Juhee Cho Sun-Young Kong Kui Son Choi Eun Sook Lee https://orcid.org/0000-0003-2166-8555 https://orcid.org/0000-0002-2410-6120 https://orcid.org/0000-0001-7979-7419 https://orcid.org/0000-0001-7750-4213 https://orcid.org/0000-0001-9081-0266 https://orcid.org/0000-0003-0620-4058 https://orcid.org/0000-0001-5336-3874 https://orcid.org/0000-0003-1122-8230

\section{REFERENCES}

1. Collins FS, Varmus H. A new initiative on precision medicine. N Engl J Med 2015;372:793-5.

2. Larry Jameson J, Longo DL. Precision medicine-personalized, problematic, and promising. Obstet Gynecol Surv 2015;70:612-4.

3. Mirnezami R, Nicholson J, Darzi A. Preparing for precision medicine. N Engl J Med 2012;366:489-91.

4. Ashley EA. The precision medicine initiative: a new national effort. JAMA 2015;313:2119-20.

5. National Library of Medicine (US). What is the precision medicine initiative? [accessed on 2018 November 5]. Available at: https:// ghr.nlm.nih.gov/primer/precisionmedicine/initiative.

6. Keogh B. Personalised medicine strategy: NHS England board [ac- cessed on 2018 November 7]. Available at: https://www.england. nhs.uk/wp-content/uploads/2015/09/item5-board-29-09-15.pdf.

7. Lévy Y. Genomic medicine 2025: France in the race for precision medicine. Lancet 2016;388:2872.

8. Asano H. Personalized and precision medicine in Japan. Asia Health Policy Program working paper \#43. Stanford (CA): Stanford University; 2017.

9. Cyranoski D. China embraces precision medicine on a massive scale. Nature 2016;529:9-10.

10. The Ministry of Health and Welfare. Promoting personalized medical care and securing future growth engines through the Ministry of Health and Welfare and Precision Medicine [accessed on 2018 November 12]. Available at: http://www.mohw.go.kr/react/al/ sal0301vw.jsp?PAR_MENU_ID=04\&MENU_ID=0403\&page $=1 \&$ CONT_SEQ=333832.

11. Moon S, Jang K, Kim H. Success strategy of precision medicine. KISTEP InI 2016;15:14-32.

12. Johnson RB, Onwuegbuzie AJ. Mixed methods research: a research paradigm whose time has come. Educational Researcher 2004; 33:14-26.

13. Juengst E, McGowan ML, Fishman JR, Settersten RA Jr. From "personalized" to "precision" medicine: the ethical and social implications of rhetorical reform in genomic medicine. Hastings Cent Rep 2016;46:21-33.

14. Bonter K, Desjardins C, Currier N, Pun J, Ashbury FD. Personalised medicine in Canada: a survey of adoption and practice in oncology, cardiology and family medicine. BMJ Open 2011;1:e000110.

15. Stanek EJ, Sanders CL, Taber KA, Khalid M, Patel A, Verbrugge RR, et al. Adoption of pharmacogenomic testing by US physicians: results of a nationwide survey. Clin Pharmacol Ther 2012;91:450-8.

16. Mountcastle-Shah E, Holtzman NA. Primary care physicians' perceptions of barriers to genetic testing and their willingness to participate in research. Am J Med Genet 2000;94:409-16.

17. Vorderstrasse A, Katsanis SH, Minear MA, Yang N, Rakhra-Burris T, Reeves JW, et al. Perceptions of personalized medicine in an academic health system: educational findings. J Contemp Med Educ 2015;3:14-9.

18. Pritchard DE, Moeckel F, Villa MS, Housman LT, McCarty CA, McLeod HL. Strategies for integrating personalized medicine into healthcare practice. Per Med 2017;14:141-52.

19. McGrath S, Ghersi D. Building towards precision medicine: empowering medical professionals for the next revolution. BMC Med Genomics 2016;9:23.

20. NHS Scotland. Scottish Medicines Consortium [accessed on 2018 November 20]. Available at: http://www.scottishmedicines.org. uk/Home. 